\title{
Correction to: Synthesis, characterization, and fluorescence study of tryptone-self-assembled and Zn(II)-coordinated nanoparticles
}

\author{
Shahzad Anwar ${ }^{1,5} \cdot$ Xiang Zhang ${ }^{1,3,4} \cdot$ Wei $\mathrm{Ji}^{2,3,4} \cdot$ Xiaolan Xu ${ }^{1}$
}

Published online: 9 April 2021

(c) King Abdulaziz City for Science and Technology 2021

Correction to: Applied Nanoscience (2020) 10:1571-1580
https://doi.org/10.1007/s13204-020-01262-9

In the published article, the corresponding author "Shahzad Anwar" has missed to include the current affiliation and it is given below.

Shahzad Anwar ${ }^{1,5}$

5. University of Chinese Academy of Sciences, Beijing 100,049, China

Publisher's Note Springer Nature remains neutral with regard to jurisdictional claims in published maps and institutional affiliations.

The original article can be found online at https://doi.org/10.1007/ s13204-020-01262-9.

Shahzad Anwar

sanwar@ibp.ac.cn

$\triangle$ Xiaolan Xu

xiaolanxu@ibp.ac.cn

1 National Laboratory of Biomacromolecules, CAS

Centre for Excellence in Biomacromolecules, Institute

of Biophysics, Chinese Academy of Sciences,

Beijing 100101, China

2 College of Life Science, University of Chinese Academy of Sciences, Beijing 100049, China

3 Physical Science Laboratory, Huairou National Comprehensive Science Center, Beijing 101400, China

4 Center for Biological Instrument Development, Core Facility for Protein Research, Institute of Biophysics, Chinese Academy of Sciences, Beijing 100101, China

5 Present Address: University of Chinese Academy of Sciences, Beijing 100049, China 